\title{
Clinical spectrum of uncomplicated malaria in semi-immune Amazonians: beyond the "symptomatic" vs "asymptomatic" dichotomy
}

\author{
Mônica da Silva-Nunes ${ }^{+}$, Marcelo U Ferreira \\ Departamento de Parasitologia, Instituto de Ciências Biomédicas, Universidade de São Paulo, \\ Av. Prof. Lineu Prestes 1374, 05508-900 São Paulo, SP, Brasil
}

We analyzed prospectively 326 laboratory-confirmed, uncomplicated malarial infections (46.3\% due to Plasmodium vivax, $35.3 \%$ due to $\mathrm{P}$. falciparum, and $18.4 \%$ mixed-species infections) diagnosed in 162 rural Amazonians aged 5-73 years. Thirteen symptoms (fever, chills, sweating, headache, myalgia, arthralgia, abdominal pain, nausea, vomiting, dizziness, cough, dyspnea, and diarrhea) were scored using a structured questionnaire. Headache (59.8\%), fever (57.1\%), and myalgia (48.4\%) were the most frequent symptoms. Ninety-six (29.4\%) episodes, all of them diagnosed during cross-sectional surveys of the whole study population (96.9\% by molecular technique only), were asymptomatic. Of 93 symptom-less infections left untreated, only 10 became symptomatic over the next two months following diagnosis. Fever was perceived as "intense" in $52.6 \%$ of 230 symptomatic malaria episodes, with no fever reported in $19.1 \%$ episodes although other symptoms were present. We found significant differences in the prevalence and perceived intensity of fever and other clinical symptoms in relation to parasite load at the time of diagnosis and patient's age, cumulative exposure to malaria, recent malaria morbidity, and species of malaria parasite. These factors are all likely to affect the effectiveness of malaria control strategies based on active or passive detection of febrile subjects in semi-immune populations.

Key words: malaria - clinical manifestations - clinical immunity - Amazonia

Despite several decades of intensive control efforts, malaria remains a leading cause of morbidity in Brazil (Loiola et al. 2002), with over 600,000 slide-confirmed cases recorded countrywide in 2005 (Ministry of Health of Brazil, unpublished data). Across the country's main endemic area, the Amazon Basin, a widespread network of outposts provides free malaria diagnosis (based on thick smear microscopy) and free treatment of slideconfirmed malaria episodes (with standardized drug regimens). Nearly all infected subjects are identified through either active or passive case detection (ACD and PCD, respectively).

Both ACD and PCD rely on the presence of symptoms - more specifically, of fever - to diagnose malarial infections. Cases are found through PCD when febrile subjects attending any health facility have a blood sample tested positive for malaria parasites; ACD implies periodic visits to households in endemic areas, with collection of thick blood smears from every person having had fever since the last visit (Nájera 2001). Aggressive active case detection (AACD), which relies on mass surveys of the whole population of a given endemic area, irrespective of any clinical symptoms, is currently limited to a few settings of very high endemicity in Brazil, such as the Yanomami Indian Reservation (Macauley 2005).

Financial support: Fapesp, grants 03/09719-6 and 04/00373-2, CNPq, grant 470067/2004-7. MdSN and MUF are Fapesp and CNPq research fellows, respectively.

+Corresponding author: msnunes1@yahoo.com.br

Received 16 February 2007

Accepted 16 April 2007
A major limitation of ACD- and PCD-based control strategies is that asymptomatic infections go undetected and untreated (Macauley 2005, Coura et al. 2006) and may represent a significant source of gametocytes for local vectors (Alves et al. 2005). There is another major limitation, however, that has been rarely addressed: the variation in the clinical expression of symptomatic malaria in semi-immune populations, ranging from a very mild, oligosymptomatic illness to the full-blown disease with periodic paroxysms described in textbooks (Fairhust $\&$ Wellems 2005). After years of exposure to malaria, many subjects tend to have milder symptoms when infected, although sterilizing immunity is rarely observed (Fairhust \& Wellems 2005). Nearly all nonimmune travelers with malaria attending infectious disease clinics in Germany (Jelinek et al. 1994) and Australia (Elliot et al. 2004) report fever, but its prevalence in semi-immune Amazonians under ACD- or PCD-based surveillance remains unknown. Here we describe the clinical expression of uncomplicated malaria episodes diagnosed during a 15-month prospective cohort study in rural Amazonia that combined ACD, PCD, and AACD.

\section{SUBJECTS AND METHODS}

Study area and population - We studied prospectively 326 malaria episodes diagnosed through AACD, $\mathrm{ACD}$, and PCD during the first 15 months of follow-up (March 2004-May 2005) of an ongoing, populationbased descriptive cohort study in Ramal do Granada $\left(9^{\circ} 41-9^{\circ} 49^{\prime} \mathrm{S}, 67^{\circ} 05 \mathrm{~W}-67^{\circ} 07^{\prime} \mathrm{W}\right)$, in the eastern part of the state of Acre, Northwestern Brazil (Silva-Nunes et al. 2006). Both P. falciparum and $P$. vivax are transmitted year-round. All households in the area were visited by our research team; because only subjects aged five years or older were eligible for blood collection during cross-sectional surveys and could provide more accurate informa- 
tion about clinical symptoms, this analysis is restricted to subjects in this age group ( $83.5 \%$ of the study population).

Baseline information - A baseline questionnaire was applied to all study participants at enrolment, to obtain demographic, clinical, and socioeconomic information and to assess their cumulative exposure to malaria. Since most adults were migrants from malaria-free areas, their ages do not necessarily correlate with exposure to malaria; this was estimated as the length of residence in malaria-endemic areas, either in Acre or elsewhere in Amazonia. The number of slide-confirmed malaria episodes recorded in local malaria diagnosis outposts between January 2001 and March 2004 was used as a proxy of recent malaria morbidity.

$A A C D$ - Three cross-sectional surveys of the whole study population aged five years or older were carried in March-April 2004, September-October 2004, and February-March 2005. Subjects were enrolled for clinical examination and blood collection irrespective of any clinical symptoms. Of 405 eligible subjects present in the study area during the first mass survey, 388 (95.8\%) and $386(95.3 \%)$ were examined for malaria parasites by thick smear microscopy and polymerase chain reaction (PCR), respectively. Of 411 subjects eligible for the second mass survey, $378(92.0 \%)$ and $379(92.2 \%)$ were examined for malaria parasites by thick-smear microscopy and PCR, respectively. Finally, 329 (86.6\%) and $328(86.3 \%)$ of 380 eligible subjects were examined for malaria parasites by thick-smear microscopy and PCR, respectively, during the last survey.

$A C D$ and PCD - Both ACD- and PCD-based surveillance of symptomatic malaria were performed in the study area between March 2004 and May 2005. For ACD, a study clinician visited the area five days/week (MondayFriday) and examined all study participants reporting recent fever, headache or any other symptom that they thought as being possibly associated with malaria. Venous or finger-prick blood samples were obtained for malaria diagnosis by microscopy and PCR. For PCD, all new patient charts kept at the three local malaria diagnosis outposts were examined daily to detect additional malaria episodes among study participants who reported directly to these facilities. Whenever possible, pre-treatment venous or finger-prick blood samples were obtained from PCD patients to confirm microscopy results and to perform PCR. A minimum interval of 28 days between two or more consecutive samples was required to count the second positive slide or PCR result as a new malaria episode; when different species were detected in samples obtained less than 28 days apart, the subject was considered to have a single episode of mixed-species infection.

Laboratory diagnosis of malaria - Thick blood smears were stained with Giemsa, and at least 100 fields were examined under $700 \times$ magnification for malaria parasites by two experienced local microscopists. Three sets of slides were sent for review by an expert microscopist at the National Reference Laboratory of the Ministry of Health of Brazil: (a) all positive slides, (b) those with discordant results, and (c) those collected from febrile subjects, but with negative results. After review, parasite loads were stratified into three levels: (a) negative slide, $(\mathrm{b}) \leq 100$ parasites in 100 fields (roughly corresponding to 1-300 parasites/ $\mu$ l of blood), and (c) > 100 parasites in 100 fields (> 300 parasites/ $\mu$ l of blood). Blood samples collected through AACD, ACD, and PCD were also examined by nested PCR-based amplification of a species-specific segment of the $18 S$ rRNA gene of human malaria parasites, as described by Kimura et al. (1997) with the modifications introduced by Win et al. (2002). DNA templates for PCR amplification were isolated from $200 \mu \mathrm{l}$ of whole venous blood, when available, using GFX genomic blood DNA purification kits (Amersham Pharmacia Biotech, Piscataway, US). DNA templates from finger-prick blood samples spotted onto FTA Micro Cards (Whatman, Clifton, US) were prepared as recommended by the manufacturer, using the FTA Purification Reagent (Whatman). Subjects with positive PCR results but negative thick-smear microscopy were considered to harbor subpatent parasitemias $(<10$ parasites/ $\mu$ l of blood).

Clinical assessment - Symptoms associated with malaria episodes, confirmed by microscopy or PCR in our cohort subjects aged five years or more, were prospectively assessed as described by Karunaweera et al. (1998). Briefly, a semiquantitative structured questionnaire addressing 13 common symptoms (fever, chills, sweating, headache, myalgia, arthralgia, abdominal pain, nausea, vomiting, dizziness, cough, dyspnea, and diarrhea) was applied to all patients. The same medical doctor (MdSN) assessed all infections, to minimize interobserver variation. According to the patient's perception, each clinical manifestation (except for fever) was considered to be absent, mild, moderate or severe; fever was classified as absent, mild or severe. Numerical scores of $0,1,2$ or 3 were assigned to symptoms reported to be absent, mild, moderate or severe, respectively. To minimize recall bias, patients were interviewed during the acute malaria episode or up to one week after treatment. Severe malaria episodes, according to the definition of the World Health Organization (2000), were not diagnosed in our study population during the follow-up, although three patients $(0.9 \%)$ required hospitalization to receive intravenous antimalarial drugs.

Treatment of malaria episodes - Malaria infections diagnosed by onsite microscopy were treated according to the current malaria therapy guidelines in Brazil (Fundação Nacional de Saúde 2001). P. vivax episodes were treated with chloroquine $(25 \mathrm{mg} / \mathrm{kg}$ of base over three days; maximum adult dose, $1.5 \mathrm{~g}$ over three days) plus primaquine $(0.5 \mathrm{mg} / \mathrm{kg} /$ day of base for seven days; maximum adult dose, $30 \mathrm{mg} /$ day). Primaquine was not given to infants $\leq 6$ months of age, pregnant and breastfeeding women or subjects with severe glucose6-phosphate dehydrogenase deficiency, which was diagnosed using the colorimetric method of Tantular and Kawamoto (2003). P. falciparum episodes were treated with mefloquine $(15 \mathrm{mg} / \mathrm{kg}$ of base, single dose; maximum adult dose, $1000 \mathrm{mg})$ or quinine $(30 \mathrm{mg} / \mathrm{kg} /$ day of salt for three days; maximum adult dose, $2000 \mathrm{mg} /$ day) plus doxycycline $(3.3 \mathrm{mg} / \mathrm{kg} /$ day of salt, for 7 days, maximum adult dose, $200 \mathrm{mg} /$ day). 
Statistical analysis - Symptom scores were compared across groups with the nonparametric tests of Mann-Whitney (two groups) and Kruskal-Wallis (more than two groups). We used the Somers' $d$ statistic (Somers 1962) to test for a dose-response relationship between symptom scores and other ordinal variables (levels of parasitemia, age, time of residence in Amazonia, and number of recent malaria episodes); the last three variables were stratified into quartiles for this purpose. Analyses were made with SPSS 13.0 (SPSS Inc., Chicago, US). Statistical significance was defined at the 5\% level.

Ethical clearance - Approval of the study protocol was obtained from the Ethical Review Board of the Institute of Biomedical Sciences of the University of São Paulo, Brazil (318/2002 and 538/2004). All study participants provided written informed consent.

\section{RESULTS}

Five hundred and nine residents $(98.5 \%$ of the eligible population), with ages ranging between one day and 90 years, were enrolled between March and September 2004. The mean incidence of symptomatic $P$. falciparum and $P$. vivax episodes confirmed by microscopy was $1.39 / 100$ person-months at risk and $2.67 / 100$ personmonths at risk, respectively, during the follow-up.

The prevalence and perceived severity of 13 symptoms were analyzed in 326 consecutive malaria episodes (found through AACD, ACD, and PCD and confirmed by thick-smear microscopy and/or PCR). Such episodes occurred in 162 subjects aged 5-73 years [mean, 24.4 years; standard deviation (SD), 16.3 years] (Fig. 1A), with an average of $2.0(\mathrm{SD}, 1.1)$ episodes per subject (range, 1-6 episodes). This sample includes $151 P$. vivax infections $(46.3 \%), 115$ P. falciparum infections (35.3\%), and 60 mixed-species (P. vivax and P. falciparum) infections (18.4\%); parasites in $135(41.4 \%)$ episodes were found by PCR only. Only $14(8.6 \%)$ subjects reported no previous slide-confirmed malaria episode, suggesting that most of them had already developed some degree of clinical immunity to malaria parasites. Headache $(59.8 \%)$, fever $(57.1 \%)$, and myalgia $(48.5 \%)$ were the most frequent symptoms reported; 96 (29.4\%) episodes were asymptomatic at the time of blood collection.

Fig. 1B shows the perceived symptom severity assessed during 230 symptomatic malaria episodes diagnosed in 129 subjects. This sample includes 122 P. vivax infections, 73 P. falciparum infections, and 35 mixedspecies infections; 34 (14.8\%) episodes were diagnosed during the cross-sectional surveys and the remaining through ACD- and PCD-based surveillance; 42 (18.3\%) were diagnosed by PCR only. Again, headache ( $84.8 \%$ ), fever $(80.9 \%)$, and myalgia $(68.7 \%)$ were the most frequent symptoms.

Ninety-nine study participants $(61.1 \%)$ reported symptoms during all infections diagnosed during the follow-up (range, 1-6 symptomatic episodes per subject), while 30 subjects (18.5\%) had both symptomatic and asymptomatic infections and 33 (20.4\%) had only asymptomatic infections. All symptom-less infections (29 with $P$. vivax, 42 with $P$. falciparum, and 25 with
A

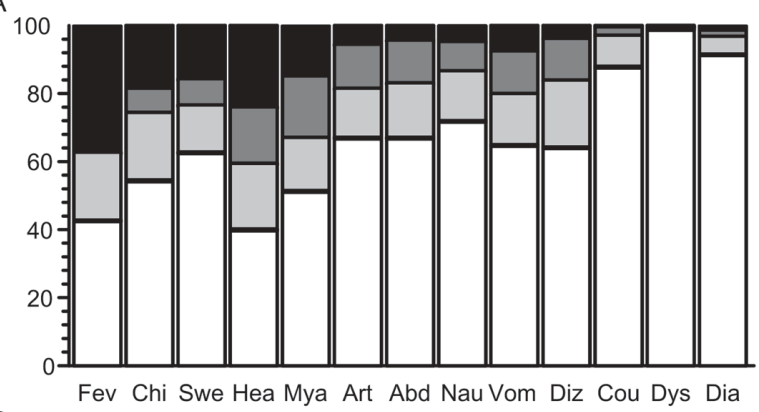

B



Fig. 1: perceived severity of 13 symptoms during 326 uncomplicated malarial infections in 162 rural Amazonians, 2004-2005. Symptoms are abbreviated as follows: fever: Fev, chills: Chi, sweating: Swe, headache: Hea, myalgia: Mya, arthralgia: Art, abdominal pain: Abd, nausea: Nau, vomiting: Vom, dizziness: Diz, cough: Cou, dyspnea: Dys, diarrhea: Dia. The shading pattern of each bar segment indicates the proportion of patients reporting a given symptom as absent (no shading), mild (light gray), moderate (dark gray) or severe (black). Numbers on y-axis are percentages. A: results for all 326 episodes, irrespective of the presence of symptoms at the time of blood collection; B: results for 230 episodes, observed in 129 subjects, during which at least one of the above mentioned symptoms was reported at the time of blood collection. All infections were laboratory-confirmed (thick-smear microscopy or polymerase chain reaction).

both species) were detected by AACD during three crosssectional surveys carried out at six-month intervals; with three exceptions (one $P$. falciparum and two mixed-species infections detected by microscopy and therefore treated), all other asymptomatic infections were missed by conventional thick-smear microscopy onsite and were left untreated because PCR results were not available timely. Subjects who had only symptom-less infections $(\mathrm{n}=33)$ did not differ significantly from those with symptomatic infections only $(\mathrm{n}=99)$ according to age (mean, 27.3 vs 22.8 years, $P=0.405$, Mann-Whitney test) or time of residence in Amazonia (mean, 16.2 vs 12.4 years, $P=0.098$, Mann-Whitney test), but had a lower number of slide-confirmed symptomatic malaria episodes documented between January 2001 and March 2004 (mean, 0.36 vs 0.66 episodes, $P=0.003$, MannWhitney test).

To determine whether asymptomatic infections detected by PCR only will eventually result, if untreated, in symptomatic disease detectable by ACD or PCD, we reviewed all symptomatic malaria episodes diagnosed in our cohort during two months following each crosssectional survey to check for the occurrence of symptoms in a subject with a previous diagnosis of asymp- 
tomatic malaria. Only $10(10.7 \%)$ of the 93 symptomless infections detected by PCR only became symptomatic over the subsequent two months of follow-up.

Most symptoms were more frequent and severe with increasing parasite loads (Fig. 2). There was a significant dose-response relationship between symptom scores and levels of parasitemia, except for cough and dyspnea (that were relatively infrequent in the patient population); $P$ values of the Somers' $d$ statistic ranged between 0.044 (diarrhea) and $<0.0001$ (other symptoms). We next compared symptom scores in infections with low (1-300 parasites/ $\mu$ l of blood; $n=53)$ or moderate to high (>300 parasites/ $\mu$ l of blood, $n=138$ ) parasitemias detected by thick-smear microscopy (Figs 2B,C respectively). Fever $(P=0.002)$, chills $(P<$ $0.0001)$, sweating $(P=0.002)$, and myalgia $(P=0.032)$ were significantly more severe in infections with mod-

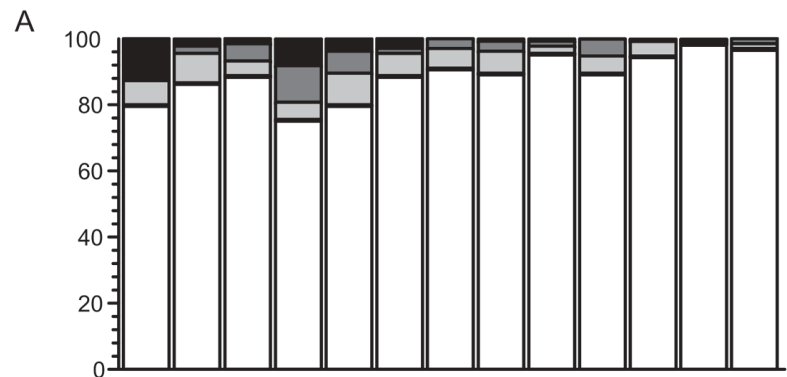

B


C

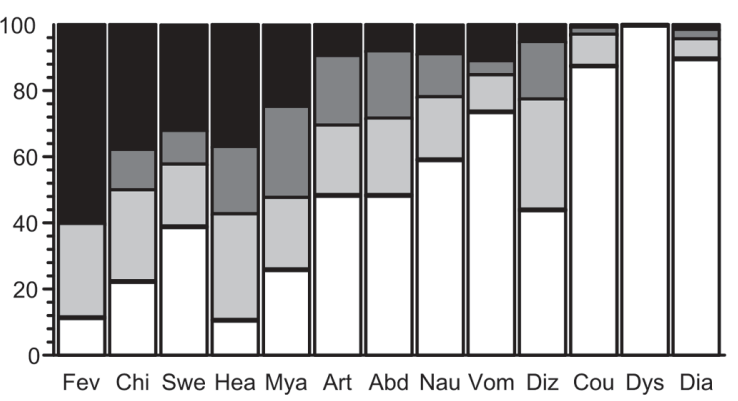

Fig. 2: perceived severity of 13 symptoms during 326 uncomplicated malaria episodes (either symptomatic or not) in 162 rural Amazonians, according to the parasite loads at the time of diagnosis. Symptoms abbreviations and bar shading patterns are as in Fig. 1. Numbers on y-axis are percentagens. A: results for infections with subpatent parasitemias $(<10$ parasites $/ \mu 1$ of blood) detected only by polymerase chain reaction $(n=135)$; B: results for infections with low but patent parasitemias (10-300 parasites/ $\mu 1$ of blood) $(n=53)$; : results for infections with the highest parasite burdens (> 300 parasites/ $\mu$ l of blood) $(n=138)$. The proportion of asymptomatic infections at the time of blood collection was $62.2 \%$ in plot A, $7.5 \%$ in B, and $0.0 \%$ in $\mathrm{C}\left(P<0.0001, \chi^{2}\right.$ test for trend $)$. erate to high parasitemia when compared with those with low parasitemia (Mann-Whitney test).

Data shown in Fig. 3 suggest that most symptoms tend to be more severe in $P$. vivax-only infections than in $P$. falciparum or mixed-species infections. In fact, KruskalWallis tests detected significant differences in the scores of fever $(P=0.005)$, chills $(P<0.0001)$, sweating $(P=$ $0.034)$, headache $(P<0.001)$, myalgia $(P=0.001)$, arthralgia $(P=0.026)$, nausea $(P=0.024)$, and dizziness $(P=0.023)$, when comparing $P$. vivax $(\mathrm{n}=151), P$. falciparum $(\mathrm{n}=115)$ and mixed-species infections $(\mathrm{n}=$ 60). Pair-wise comparisons with the Mann-Whitney test revealed that these differences were due to higher scores in the $P$. vivax group.

To explore these findings, we next compared symptom scores in $P$. vivax-only and $P$. falciparum-only infections after stratifying the infections into two levels

A
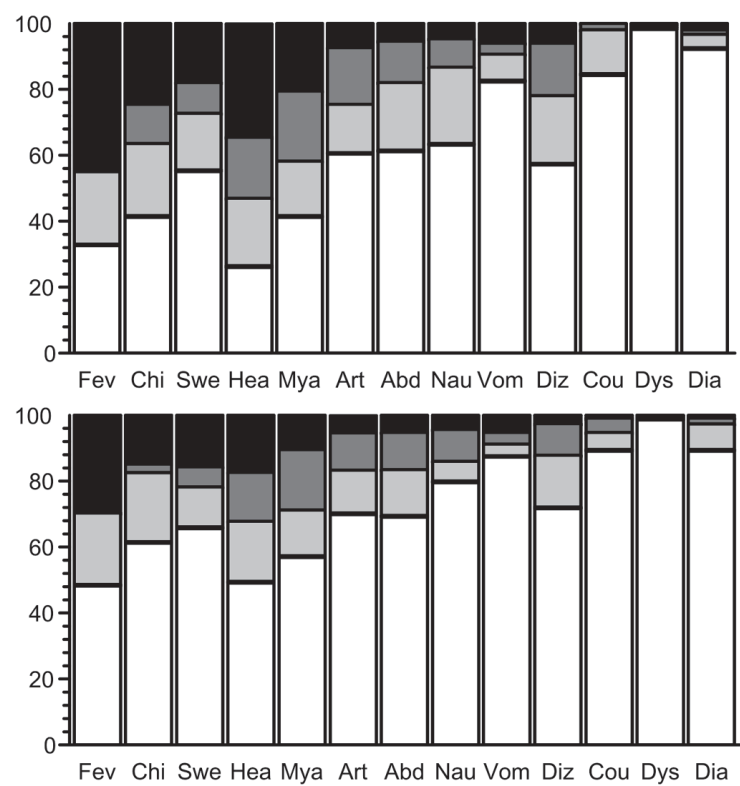

C

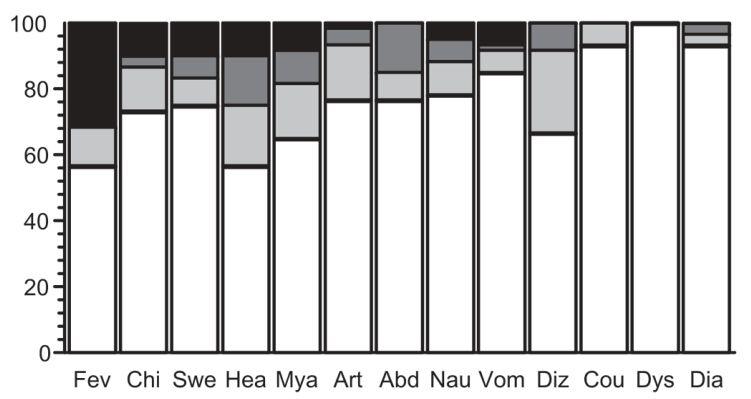

Fig. 3: perceived severity of 13 symptoms during 336 uncomplicated malaria episodes in 162 rural Amazonians, according to the species of infecting parasites. Symptoms abbreviations and bar shading patterns are as in Fig. 1. Numbers on y-axis are percentagens. A: results for infections with Plasmodium vivax only $(\mathrm{n}=151)$; B: results for infections with P. falciparum only $(\mathrm{n}=115) ; \mathrm{C}$ : results for mixed-species infections $(\mathrm{n}=$ 60). The proportion of asymptomatic infections at the time of blood collection are $18.5 \%$ in plot A, $37.4 \%$ in B, and $41.7 \%$ in $\mathrm{C}\left(P<0.0001, \chi^{2}\right.$ test). All infections were laboratory-confirmed (thick-smear microscopy or polymerase chain reaction). 
of parasitemia (1-300 parasites/ $\mu 1$ and $>300$ parasites/ $\mu 1$ ); episodes with low parasitemias detected only by PCR were excluded, because $62.2 \%$ of them were asymptomatic. Stratification revealed that most differences were likely to be due to confounding, since $P$. falciparum infections in our sample were often associated with very low parasitemias. In other words, $P$. falciparum infections are overrepresented in the group with light parasite burdens that tend to be less symptomatic. Within each level of parasitemia, however, symptom scores were quite similar between species; significant differences (Mann-Whitney test) were found only for headache (more severe in $P$. vivax than in $P$. falciparum infections, among those with 1-300 parasites/ $\mu$ l of blood, $P$

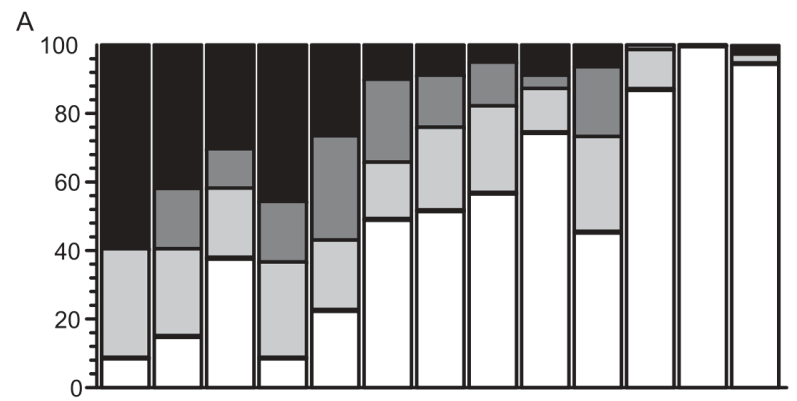

B

Fev Chi Swe Hea Mya Art Abd Nau Vom Diz Cou Dys Dia

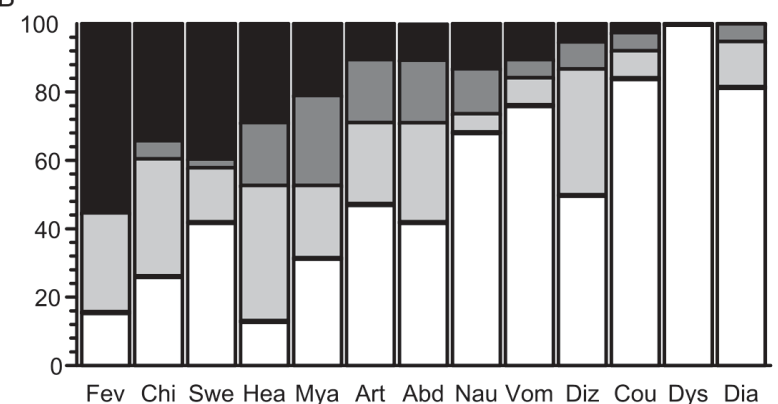

Fig. 4: perceived severity of 13 symptoms during 117 uncomplicated malaria episodes with the highest parasite burdens (parasitemia $>300$ parasites/ $\mu$ l of blood), according to the species of infecting parasites, 2004-2005. Symptoms abbreviations and bar shading patterns are as in Fig. 1. Numbers on y-axis are percentagens. A: results for infections with Plasmodium vivax only $(\mathrm{n}=79)$; B: results for infections with $P$. falciparum only $(\mathrm{n}=38)$.
$=0.017$ ) and diarrhea (more severe in P. falciparum than in $P$. vivax infections, among those with $>300$ parasites/ $\mu 1$ of blood, $P=0.025$ ) (Fig. 4).

We next examined whether some malaria symptoms tended to become milder with increasing age and levels of cumulative exposure to malaria. Ninety-two (56.8\%) of our 162 patients had more than one malarial infection diagnosed between March 2004 and May 2005; in these cases, we considered only the symptoms associated with the first episode occurring during the followup. Only fever became less frequent and less severe with increasing age and time of residence in Amazonia (Table). Subjects with a greater number of recent slide-confirmed symptomatic malaria episodes (recorded during the 39 months preceding the study) had infections that were significantly more symptomatic during the cohort follow-up (Table).

\section{DISCUSSION}

Asymptomatic malarial infection and full-blown illness with typical febrile paroxysms are extreme points in the wide spectrum of clinical disease caused by malaria parasites. Naturally acquired clinical immunity, parasite load and virulence, and host's age and genetic factors are all likely to modulate, in different ways, the clinical expression of malaria. This originates intermediate levels of disease severity that do not fit into dichotomic ("all-or-nothing") categories such as "asymptomatic" vs "symptomatic" infection. Therefore, ACDand PCD-based strategies of malaria control, such as those currently used in Brazil, must deal with a heterogeneous disease in which high fever and cyclical paroxysms with chills and profuse sweating, the hallmark of textbook malaria, are not necessarily prominent features. For example, only $52.6 \%$ of 230 episodes of laboratory-confirmed symptomatic malaria diagnosed during our prospective study in rural Amazonia were associated with fever perceived as "intense"; no fever was reported in $19.1 \%$ of such episodes, although other symptoms were present (Fig. 1B) and most subjects felt ill enough to seek malaria diagnosis through ACD or PCD. In fact, most afebrile patients and those with low parasitemia had thick smears examined because of either persistent or intermittent headache or myalgia, which highlights the importance of a broader clinical surveillance.

TABLE

Variation in the perceived intensity of symptoms during 162 malaria episodes diagnosed in a cohort of rural Amazonians, according to increasing age, cumulative exposure to malaria and recent morbidity, 2004-2005

Variable

(stratified into quartiles)

Age

Time of residence in Amazonia

No. of recent slide-confirmed malaria episodes ${ }^{a}$

$$
\begin{aligned}
& \text { Change in symptom intensity scores } \\
& (P \text { value, Somers' } d \text { test for ordinal variables }) \\
& \downarrow \text { fever }(P=0.035), \uparrow \text { myalgia }(P=0.009), \\
& \uparrow \text { arthralgia }(P<0.0001) \\
& \downarrow \text { fever }(P=0.014), \uparrow \text { abdominal pain }(P=0.021) \\
& \uparrow \text { chills }(P=0.004), \uparrow \text { sweating }(P=0.020), \uparrow \text { headache } \\
& (P=0.031), \uparrow \text { arthralgia }(P<0.0001), \uparrow \text { dizziness }(P=0.016)
\end{aligned}
$$

A single episode was analyzed per subject; for those with more than one malaria episode between March 2004 and May 2005, only the first episode diagnosed during the follow-up was considered. Only significant results (Somers' $d$ statistic, $P<0.05$ ) are shown; $a$ : malaria episodes diagnosed through active and passive case detection between January 2001 and March 2004. 
Are clinical manifestations different in nonimmune and semi-immune patients with symptomatic but uncomplicated disease? Although the clinical presentation of imported malaria has been described in detail in several recent studies (reviewed by Elliot et al. 2004), most case series include heterogeneous mixtures of nonimmune travelers and semi-immune expatriates and foreign visitors, with varying age and geographic origin, precluding meaningful comparisons. Not surprisingly, nearly all nonimmune travelers report fever (Jelinek et al. 1994, Elliot et al. 2004), while this symptom tends to be less prevalent (79-98\%) in case series including putatively semi-immune expatriates and foreign visitors, in addition to nonimmune travelers (Svenson et al. 1995, Dorsey et al. 2000, Sanchez et al. 2000). However, comparisons of semi-immune and nonimmune patients in the same case series found no significant differences in the prevalence of selected symptoms (Svenson et al. 1995) and hospitalization rates (Dorsey et al. 2000), although no attempt was made to quantify the perceived severity of symptoms in a standardized way. Further comparisons across studies are hampered by the use of different ways of determining and reporting the prevalence and intensity of each symptom or sign and by the inclusion of subjects with different ages who were infected with different malaria parasite species.

Is there any association between the prevalence or perceived intensity of symptoms and the malaria parasite species found in uncomplicated disease? Diarrhea was found to be significantly more frequent in $P$. falciparum than in $P$. vivax infections in two large case series (Svenson et al. 1995, Robinson et al. 2001), and in the semi-immune Amazonians in this study with the highest parasitemias (Fig. 4). Temperatures greater than $39^{\circ} \mathrm{C}$ (Svenson et al. 1995) and rigors (Robinson et al. 2000) have been reported to occur more commonly in $P$. vivax infections, while drowsiness was found more frequently in $P$. falciparum infections (Robinson et al. 2000), but these differences have not been independently confirmed in other populations.

We found significant differences in the prevalence and perceived intensity of fever and other clinical symptoms in relation to parasite load at the time of diagnosis and patient's age and levels of cumulative exposure to malaria and recent malaria morbidity, all of which may affect the sensitivity of ACD and PCD strategies in semiimmune populations. As a consequence, infections may remain undetected in a substantial proportion of subjects with absent or atypical clinical manifestations (such as exclusive mild to moderate headache). These undetected infections have been suggested to represent a significant source of parasites for local mosquitoes, maintaining residual malaria transmission (Coura et al. 2006).

Although fever may become less intense with increasing age and time of residence in Amazonia, myalgia and arthralgia are perceived as more severe by older subjects, while abdominal pain is perceived as more severe by subjects with a longer stay in endemic areas (Table). Whether increased abdominal pain is due to hepatosplenomegaly or other abdominal changes could not be assessed in this study.
Finally, are subjects presenting with symptomatic malaria more susceptible to disease or just unlucky enough to have met a more virulent parasite? We found that subjects with more episodes of symptomatic malaria documented up to 39 months before the baseline evaluation reported more intense symptoms during subsequent infections (Table). These subjects may be either more susceptible to disease manifestations or more likely to perceive their symptoms as severe and seek treatment. However, 30 (23.3\%) subjects who had laboratory-confirmed symptomatic malaria diagnosed through ACD or PCD during the follow-up were also found, during cross-sectional surveys, to be asymptomatic carriers of parasites. These findings suggest that the presence or absence of symptoms does not correspond to stable phenotypes and necessarily indicate absence or presence of clinical immunity; not surprisingly at all, a considerable proportion of current asymptomatic parasite carriers may later become ill when exposed to parasites that are intrinsically more virulent or express novel antigenic variants. To address this issue, we are currently testing whether asymptomatic parasite carriers found through AACD differ from the cohort participants with either symptomatic disease or no infection at all in the incidence of malaria morbidity during the subsequent months of follow-up.

\section{ACKNOWLEDGEMENTS}

To the inhabitants of Ramal do Granada for their enthusiastic participation in the study; Sebastião Bocalom Rodrigues (Mayor of Acrelândia), Damaris de Oliveira, and Nésio M Carvalho (Municipal Government of Acrelândia), Raimundo A Costa and the malaria control team in Acrelândia and in the Ramal do Granada, and Dr Pascoal T Muniz (Federal University of Acre) for their logistic support; Adamílson L de Souza, Dr Rosely S Malafronte, Natal S da Silva, Camila Juncansen, Carlos E Cavasini, and Kézia KG Scopel for their help in fieldwork; Estéfano A de Souza and Bruna A Luz for data management, Natália T Komatsu, Rosane R D'Arcádia, Kézia K G Scopel, and Érika M Braga for PCRbased malaria diagnosis, and Francisco das Chagas O Luz (Ministry of Health, Brasília, Brazil) for reviewing all malaria slides.

\section{REFERENCES}

Alves FP, Gil LHS, Marrelli MT, Ribolla PEM, Camargo EP, Silva LHP 2005. Asymptomatic carriers of Plasmodium spp. as infection source for malaria vector mosquitoes in the Brazilian Amazon. J Med Entomol 42: 777-779.

Coura JR, Suárez-Mutis M, Ladeia-Andrade S 2006. A new challenge for malaria control in Brazil: asymptomatic Plasmodium infection - A review. Mem Inst Oswaldo Cruz 101: 229-237.

Dorsey G, Gandhi M, Oyugi J, Rosenthal PJ 2000. Difficulties in the prevention, diagnosis, and treatment of imported malaria. Arch Intern Med 160: 2505-2510.

Elliot JH, O' Brien D, Leder K, Kitchener S, Schwartz E, Weld L, Brown GV, Kain KC, Torresi J 2004. Imported Plasmodium vivax malaria: demographic and clinical features in nonimmune travelers. J Travel Med 11: 213-219.

Fairhust RM, Wellems TE 2005. Plasmodium species (malaria). In GL Mandell, JE Bennett, R Dolin (eds), Mandell, Douglas, and Bennett's Principles and Practice of Infectious Diseases, Elsevier Churcill Livingstone, Philadelphia, p. 3121-3144. 
Fundação Nacional de Saúde 2001. Manual de Terapêutica da Malária, Ministério da Saúde, Brasília, 104 pp.

Jelinek T, Nothdurft HD, Löscher T 1994. Malaria in nonimmune travelers: a synopsis of history, symptoms, and treatment in 160 patients. J Travel Med 1: 199-202.

Karunaweera ND, Carter R, Grau GE, Mendis K 1998. Demonstration of anti-disease immunity to Plasmodium vivax malaria in Sri Lanka using a quantitative method to assess clinical disease. Am J Trop Med Hyg 58: 204-210.

Kimura M, Kaneko O, Liu Q, Zhou M, Kawamoto F, Wataya Y, Otani S, Yamaguchi Y, Tanabe K 1997. Identification of the four species of human malaria parasites by nested PCR that targets variant sequences in the small subunit rRNA gene. Parasitol Int 46: 91-95.

Loiola CC, da Silva CJ, Tauil PL 2002. Controle da malária no Brasil: 1965 a 2001. Rev Panam Salud Pública 11: 235-244.

Macauley C 2005. Aggresive active case detection: a malaria control strategy based on the Brazilian model. Soc Sci Med 60: 563-573.

Nájera JA 2001. Malaria control: achievements, problems, and strategies. Parassitologia 43: 1-89.

Robinson P, Jenney AW, Tachado M, Yung A, Manitta J, Taylor K, Biggs BA 2001. Imported malaria treated in Melbourne,
Australia: epidemiology and clinical features in 246 patients. J Travel Med 8: 76-81.

Sanchez JL, Bendet I, Grogl M, Lima JBP, Pang LW, Guimarães R, Guedes RH, Milhous WK, Green MD, Todd GD 2000. Malaria in Brazilian military personnel deployed to Angola. J Travel Med 7: 275-282.

Silva-Nunes M, Malafronte RS, Luz BA, Souza EA, Martins LC, Rodrigues SG, Chiang JO, Vasconcelos PFC, Muniz PT, Ferreira MU 2006. The Acre Project: the epidemiology of malaria and arthropod-borne virus infections in a rural Amazonian population. Cad Saúde Públ 22: 1325-1334.

Somers RH 1962. A new asymmetric measure of association for ordinal variables. Am Sociol Revi 27: 799-811.

Svenson JE, MacLean JD, Gyorkos TW, Keystone J 1995. Imported malaria: clinical presentation and examination of symptomatic travelers. Arch Intern Med 155: 861-868.

Tantular IS, Kawamoto F 2003. An improved, simple screening method for detection of glucose-6-phosphate dehydrogenase deficiency. Trop Med Int Health 8: 569-574.

Win TT, Lin K, Mizuno S, Zhou M, Liu Q, Ferreira MU, Tantular IS, Kojima S, Ishii A, Kawamoto F 2002. Wide distribution of Plasmodium ovale in Myanmar. Trop Med Int Health 7: 231-239.

World Health Organization 2000. Severe falciparum malaria. Trans $R$ Soc Trop Med Hyg 94 (Suppl. 1): S1-S90. 
